\title{
Deficit in Learning of a Motor Skill Requiring Strategy, but Not of Perceptuomotor Recalibration, with Aging
}

\author{
Ewan C. McNay ${ }^{1}$ and Daniel B. Willingham \\ Department of Psychology \\ University of Virginia \\ Charlottesville, VA 22903
}

\begin{abstract}
We investigated the effect of aging on different aspects of motor skill learning using two computer-presented perceptuomotor tasks. The relationship between visual and proprioceptive feedback was transformed in the first task, which was open to the formation and use of strategies. This task was designed to lead to perceptuomotor adaptation that was then measured by performance on a very similar second task that was not open to the use of strategy task. Older participants showed impaired learning of the strategic task but not of the nonstrategic task. This is in line with the suggestion that the effect of aging on learning and memory may be to reduce working memory resources.
\end{abstract}

\section{Introduction}

Older participants have been shown to be impaired in several motor skill learning (i.e., increasing accuracy of movement with practice; Willingham 1998) tasks when compared with younger subjects [rotor pursuit (Ruch 1934; Gutman 1965), mirror tracking and rotary pursuit (Wright and Payne 1985)], while showing unimpaired performance on one motor skill task (Howard and Howard 1989, 1992). The task used by Howard and Howard was a serial response time (SRT) task in which participants show reduced response times to successive repetitions of a sequence although they demonstrate no awareness of the existence of such a sequence. An important question,

\footnotetext{
${ }^{1}$ Corresponding author.
}

then, in studying the effects of aging on learning motor skills, is what determines whether a task is one on which older participants will show a deficit.

One might propose that the effect of aging on motor skill learning is characterized by an agelinked deficit in explicit, but not implicit, learning of motor skills (where explicit learning is learning of which the participant is aware and makes direct reference to the learning, whereas participants are unaware of implicit learning, which is typically assessed by task performance effects). Several researchers have found that performance on implicit tasks is spared by aging (e.g., Schacter 1987; Howard and Howard 1989, 1992; Craik 1994; Light et al. 1995), whereas older participants show evidence of deficits in explicit processes (e.g., Craik and Jennings 1992). However, although such a dissociation would account for the observed lack of impairment in older participants in the SRT task, it cannot account for the observed deficits in older subjects in other tasks, such as tracing objects seen only in a mirror or maintaining contact with a rotating target, which are also classed as implicit tasks. Furthermore, Harrington and Haaland (1992) used a complex stimulus-response task, similar to the SRT task in that some of the responses were sequenced, and reported a deficit for older subjects in implicit learning of the motor sequences. They concluded that the fact of a task being implicitly learned was insufficient to guarantee spared learning with aging.

In this study we investigated the possibility that the effect of aging on motor skill learning would be characterized by age-linked deficits in learning of motor skill tasks that allow the use of strategy formation. This possibility is suggested by the differences in the findings of Harrington and Haaland (1992) versus those of Howard and Howard $(1989,1992)$; it may be that the more com-

LEARNING \& MEMORY 4:411-420 @ 1998 by Cold Spring Harbor Laboratory Press ISSN1072-0502/98 \$5.00

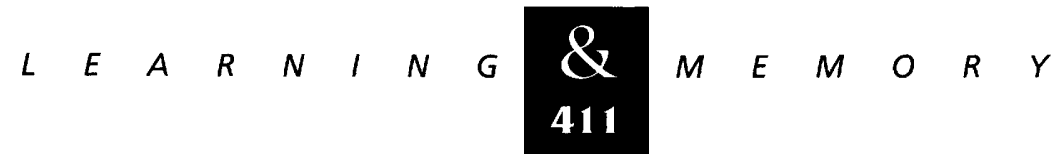


plex task used by Harrington and Haaland allowed the formation of strategies, whereas the simpler SRT task did not.

We used two perceptuomotor tasks based on those introduced by Cunningham (1985), the first of which was open to the development and use of strategies, whereas the second was not. In the first task (training phase), participants were asked to trace lines presented on a computer screen, using a stylus on a graphics tablet to manipulate the computer cursor. The relationship between movement of the stylus and movement of the cursor was transformed by the computer with a $90^{\circ}$ counterclockwise rotation. Participants were then transferred to a second, similar task (the test phase) where both the transformation and the visual feedback were removed; that is, participants were again asked to trace lines and were (correctly) told that there was no longer any transformation of the cursor's movements (i.e., that the cursor would move in the same way as the stylus), but the cursor's movements were not visible to participants (to avoid any correction of adaptation).

Consider what might affect performance in each of the two tasks. The effect of the transformation in the training phase is to place visual and proprioceptive sensory inputs in conflict. During the training task, learning might occur such that the disparity between vision and proprioception is reduced; that is, proprioception becomes recalibrated so that the disparity with visual input is reduced. Research with prism spectacles has shown that such perceptuomotor recalibration, or adaptation, does indeed occur (Redding and Wallace 1978; Welch 1978, 1986; Wallace and Redding 1979; Bedford 1993a,b). Adaptation is implicit, meaning that participants are unaware that the learning occurs and, therefore, that adaptation does not allow the development of a strategy on the part of the subject (Welch 1978; Bedford 1993a). This learning would affect performance on both training and test tasks. Following adaptation, participants will move their arms in a direction that they believe to be counterclockwise of their arm's actual position.

During the training phase, a participant might also develop a conscious strategy to improve performance; for example, a participant might mentally rotate the lines presented by $90^{\circ}$ and move his or her hand in the direction of the mentally imaged line. During the training task, learning is shown by improvement in the accuracy of participants' line tracing. Both perceptuomotor recalibration and the use of effective strategy will result in improved accuracy; strategies have been shown previously to improve performance in perceptuomotor tasks (Verdolini-Marston and Balota 1994).

In the test phase, however, strategies are inappropriate: Participants have been told that the transformation has been removed. Thus, two sources of learning may contribute to performance in the first, training task-strategy and adaptation-whereas only adaptation contributes to performance in the second task. This view of the two tasks is similar to that put forward by Redding and Wallace (1993) with regard to prism spectacle experiments, who state, "Direct effects are assumed to reflect influences of both adaptive coordination and alignment, whereas aftereffects are argued to reflect the contribution of adaptive alignment . . . Subjects have no reason to apply [during the test phase] any adaptive strategies intentionally acquired [in the training phase]." Note that both training and test phases measure learning occurring in the training phase but measure different types of learning.

We predicted that elderly participants would show deficits in strategy formation but that learning of perceptuomotor adaptation might be spared by aging. This pattern of results would be seen as a deficit in the training phase (where strategies are involved) but an absence of any age-linked effect on performance in the test phase (measuring only adaptation). Conversely, an age-linked deficit in the training phase only would show that the age-linked deficit was indeed attributable to differences in strategy formation and/or use, because that is the dimension along which the two tasks differ.

\section{Materials and Methods}

\section{PARTICIPANTS}

Fourteen young adults (mean age $=19.9$ years, S.D. $=0.9$ ) and 10 older adults (mean age $=64.2$ years, S.D. $=6.4$ ) participated. The young adults (seven females and seven males) participated to fulfill part of the course requirements for introductory psychology classes at the University of Virginia, whereas the older adults (four females and six males) volunteered from a local senior center. All participants were recruited for a "study of motor skill and aging." The education level of the two groups was comparable (young mean $=14.4$ years, S.D. $=0.48 ;$ older mean $=15.8$ years, s.D. $=3.12, t$ 
assuming unequal variances $=1.37, P>0.20$ ); note that this is sufficiently high for both groups to be highly overtrained in stylus use and that the final education level of the young participants is as yet undetermined. All participants were right-handed by self-report and had vision sufficient to clearly read 10-point type on a computer monitor at 1 meter. Samuel (1981) reported that participants with outstanding athletic or artistic talent have anomalous perceptuomotor integration, and we thus excluded from the young group any participants who, by self-report, were especially athletic or artistic. Of the older group, one female occasionally painted; her data were comparable to the group's and have been included as they alter none of the findings. All members of the older group were community dwelling and healthy by self-report.

\section{STIMULI AND APPARATUS}

Participants were tested using a Macintosh IIsi computer connected to a Wacom UD-1212-R graphics tablet and stylus. Instructions for each task were presented both on the screen (as a confirmation of visual acuity) and verbally by the experimenter, followed by a check that the instructions were clear and had been correctly understood. During both training and test phases, participants' view of their right (drawing) hand and arm was blocked by means of a wooden box around the graphics tablet that allowed free hand and arm movement, as shown in Figure 1.

Both training and test tasks consisted of being asked to trace, using the stylus, a series of straight lines presented on the monitor. Lines were of a constant length $(10.16 \mathrm{~cm})$, were clearly visible, and radiated from the center of the monitor screen in one of eight directions spaced at $45^{\circ}$ intervals from the vertical. In both phases, the cursor appeared in the center of the screen at one end of the line, and participants were told that they should immediately trace the line as accurately as possible. Participants were told that each line would be presented for $5 \mathrm{sec}$ and would then be cleared from the screen. After each line cleared, participants returned the pen to the center of the tablet, using visual guidance via a panel in the top of the wooden box. The experimenter then triggered the next line to appear. All lines were thus traced from the center of the screen out toward the perimeter. Lines were presented in random order of orientation with the constraint that each direction was

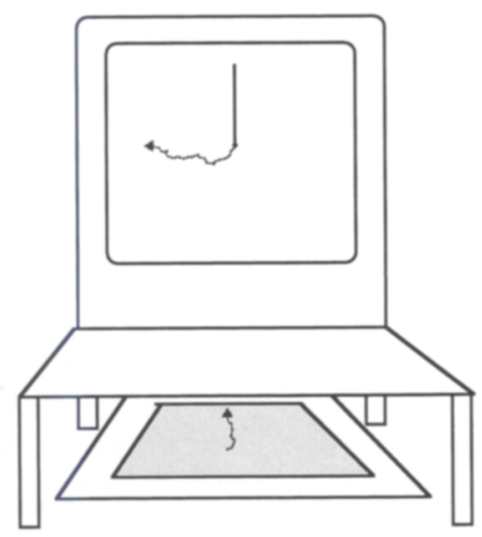

Figure 1: Apparatus used for all phases. Illustrated on the screen are a target line (straight vertical line) and the cursor trace that would be produced by an exemplar stylus trace (arrows on screen and graphics tablet, respectively) with the training phase $90^{\circ}$ counterclockwise transformation in place. The graphics tablet is blocked from the participants' view by means of a wooden box cover.

presented at least once and no more than twice in each block of 10 lines. The total number of line presentations in each direction was balanced in both phases. The use of a computer to create the perceptuomotor disparity avoided undesirable side effects of prism goggles such as blurring of vision and inappropriate curvature of the visual field, and the use of a stylus was intended to minimize any age-linked performance deficits in line tracing because participants were expected to be highly practiced in the use of a stylus. The use of a stylus should also ensure that both groups were highly overtrained in the perceptuomotor relationships being perturbed in the training phase. We hoped to thus achieve comparable baseline motor performance across age group and to avoid any confounds caused by differences between the groups in the level of pre-experiment training or skill.

During both training and test phases, the position of both pen and cursor was sampled by the computer every $50 \mathrm{msec}$, and visual feedback (during the training phase only) was provided at each sampling time via a dot ( $3 \mathrm{~mm}$ in diam.) produced on the screen at the position of the cursor. Dots remained on the screen to produce a trail. In both phases, both target line and any feedback display were cleared automatically 5 sec after initial target line presentation.

\section{PROCEDURE}

The experiment had several phases. First, base- 
line measures were taken both with and without visual feedback. In the first, baseline-feedback phase, participants were given 32 trials of practice at tracing lines; visual feedback was provided, and no transformation was applied. This phase provided a measure of baseline motor performance and line-tracing ability in the presence of feedback and confirmed that older participants did not show poor tracing performance because of motor slowness, lack of visual acuity, or other physical deficit. In the second, baseline-no-feedback phase, participants were informed that the visual feedback would now be removed and were then given 16 trials of practice at line tracing in the absence of feedback. These trials served as a second control measure of baseline line tracing ability, acclimatized participants to tracing in the absence of visual feedback, and confirmed that there was no difference with age on tracing performance in the absence of feedback.

Following these baseline phases, participants began the training phase. Participants were informed that there would now be a transformation applied to their tracing, and that this would mean that the line of dots appearing on the screen would not necessarily move in the direction in which they moved their hand. Participants were told that they should try to adjust their hand movements such that the line of dots on the screen would again follow the target line presented. The experimenter asked each participant to describe the task as a means of checking that the instructions were understood and explained the task again if necessary. No information was given as to the type of transformation that would be applied or whether it would remain constant across trials. In fact, a constant transformation (a $90^{\circ}$ counterclockwise rotation) was used for all training trials. Participants performed six blocks of training trials, with 30 trials in each block and a rest break between blocks 3 and 4.

Ten test phase trials were given after each block of 30 training trials. Participants were told that the transformation had now been removed and that they should now trace the line exactly as they had in the baseline-no-feedback phase. Participants were observed closely to ensure that they understood that for test phase trials, no transformation was being applied. Instructions for the following phase were briefly repeated before each block of either training or test trials to ensure that participants were clear on which condition was about to occur.

\section{Results}

Two types of incorrect trials were removed from the analysis: trials where the participant failed to respond correctly to the fact that transformation was no longer present on the first trial in a test block (line traced at $>45^{\circ}$ from target line), and trials where the experimenter triggered the appearance of the line before the participant was ready for it. Neither type of trial error accounted for $>1 \%$ of the data in either age group; the first type occurred only once for each of three participants, two young and one older. There was no difference between genders on any of the dependent measures (all $F \mathrm{~s}<1.2$, all $P$ s $>0.27$ ), and groups were therefore collapsed across gender.

\section{BASELINE MEASURES}

An $\alpha$ level of 0.05 was used for calculations of significance in all statistical testing. Deviation measures were calculated only on the portion of the tracing within a circle defined as having its center at the origin (the point in the center of the computer screen from which all target lines radiated) and its radius equal to the length of a target line. The measure of deviation was the average distance of each sampled cursor position from the closest point on the target line; this distance was defined to be positive when the traced line was clockwise of the target line. Signed error (i.e., deviation measures using both magnitude and direction of error) was used for both baseline phases, because both magnitude of error and any directional bias was of interest. No differences were found between the deviations of the two groups in the baseline phases (both $F<0.65$, both $P>0.40$ ). The performance of both groups was essentially perfect in the feedback condition (error means \pm S.D.: $-0.012 \pm 0.11$ and $-0.009 \pm 0.09$ for older and young participants, respectively). Errors were slightly larger, but had no significant directional bias, in the no-feedback condition (means \pm S.D.: $0.25 \pm 7.23$ and $1.52 \pm 6.77$ for older and young participants, respectively).

\section{TRAINING PHASE}

The measure of primary interest was the deviation of the line drawn from the target line. In the training phase, deviation from the target line was calculated as a mean root square distance, because the magnitude of total error was the most impor- 
tant measure. Before analyzing deviation data, mean error in the baseline-feedback phase was subtracted (independently for each line direction and each participant) from the training phase data; the same procedure was followed for test phase data using mean error scores from the baseline-nofeedback phase. This correction of data removed any effects from differences in individual ability to trace lines in each direction more or less accurately or from any existing tendency of participants to trace to one side or other of the target line. Such a correction might have been particularly important owing to possible differences in, for example, hand stability between the two age groups, although in fact no such differences were found, as noted above.

Training phase results are shown in Figure 2. As predicted, older participants showed a deficit in the training phase, with significantly greater error in tracing $[F(1,22)=20.96, \quad$ MSE $=7210$, $P<0.0001]$. Both groups showed significant learning in the training phase, shown by a significant effect of block on performance $[F(5,110)=17.16$, MSE $=673.3, P<0.0001]$. Importantly, young participants showed greater learning $[F(5,110)=4.493$, MSE $=176.33, P<0.001]$.

One possible explanation for the age-related deficit in training phase performance could have been a failure of older participants to react sufficiently quickly to trial presentation. This might result in less of a line being drawn and so less possibility for calculated error. Analysis of reaction time [(RT) defined as the time between trial presentation and first cursor movement] and length of

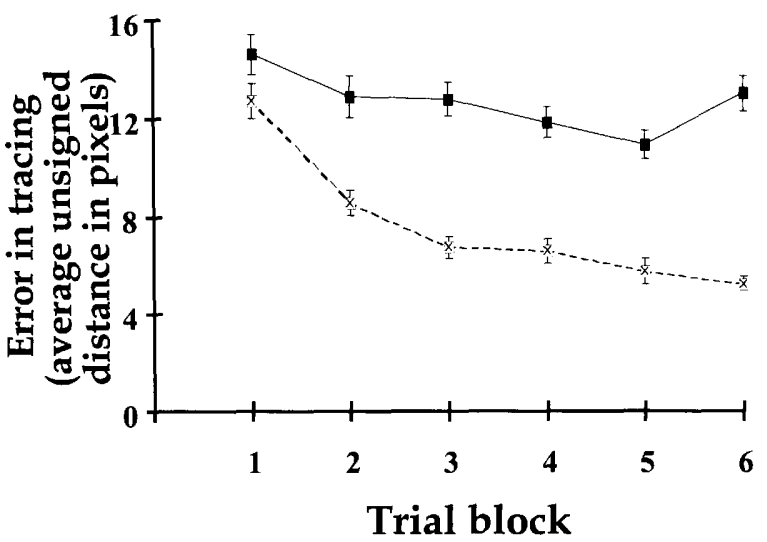

Figure 2: Mean deviation from target line for young ( $\square$ ) and older ( $\boldsymbol{\square})$ groups on the training task, by block of training trials. Deviation is measured as detailed in the text. Vertical error bars depict S.E.M.. line traced showed that this was not the case, however. In the analysis of RT data only, the data from two participants in the younger group was removed from the analysis. These participants had reaction times slower than any of the older group and $>10$ s.D.s from the mean of the remaining young participants; the inclusion of data from these participants had no effect on any finding other than those regarding RT. The effect of age on RT observed is perhaps smaller than would have been expected, given the many reports of general slowing with age (e.g., Fisk et al. 1988; Cerella 1990). Older participants showed an increase in RT (i.e., slower response) in the training phase [mean RT of $1469 \mathrm{msec}$ (older) vs. $1191 \mathrm{msec}$ (young), $F(1,20)=4.6$, M.s.E $=2712032, P<0.05$ ] Importantly, however, this increased RT did not result in the older participants tracing significantly less distance in the time available [mean distance of $7.62 \mathrm{~cm}$ (older) vs. $8.49 \mathrm{~cm}$ (young), $F(1,22)=2.64$, $\mathrm{MSE}=6527, P>0.1]$. Hence, deviation measurements are not confounded by the two groups having significantly different line lengths over which to deviate.

\section{TEST PHASE}

Deviation in the test phase was calculated as a signed error for the test task, because the direction of error in tracing was of primary interest. A positive error was defined as error in the clockwise direction from the target line, whereas error in the anticlockwise direction was given a negative sign. In the test phase, learning is observed via decreasing accuracy in tracing lines; because participants are moving their arm in a direction clockwise of its aimed position, adaptation will be shown by increased error in a clockwise direction from the target line. Learned adaptation to the training phase transformation means that participants will now have an inappropriate map between vision and proprioception, and their tracings will therefore deviate in the direction opposite to that of the transformation (Cunningham 1989). Welch (1978) called this opposite deviation the negative aftereffect.

The older group showed no difference in learning of adaptation as compared with the young group $[F(1,22)=0.58, \quad \mathrm{MSE}=96.2, P>0.8]$, as shown in Figure 3. Note that both groups show significant adaptation, indicated by consistent clockwise error. The adaptation appears to occur 
within the first 30 training trials for both participant groups, as shown by the absence of any effect of block on performance in the test phase $[F(5,110)=0.909, \mathrm{MSE}=58.11, P>0.45]$, and the absence of any interaction of test block with age group $[F(5,110)=0.276, \mathrm{MSE}=17.663, P>0.90]$. Similar considerations of RT and distance of tracing to those mentioned with respect to the training phase apply; however, there was no difference in either RT [mean RT of 1353 msec (older) vs. 1072 msec (young), $F(1,20)=4.23, \quad M S E=2632727$, $P>0.05$ ] or length of line traced [mean distance of $9.94 \mathrm{~cm} \mathrm{(older)} \mathrm{vs.} 9.79 \mathrm{~cm}$ (young), $F(1,22)=1.42, \mathrm{MSE}=119.25, P>0.2]$ across age group. The measures of RT and distance traced were of secondary interest in this study and will not be discussed further.

Line direction had a significant effect on performance in both phases: $F(7,154)=2.8, \mathrm{MSE}=152.6$, $P<0.01$ in training phase and $F(7,154)=13.06$, MSE $=3112, P<0.0001$ in test phase. As can be seen in Figure 4, however, the effect of line direction was not consistent either across age group or across phase. Although there are a variety of possible explanations for this finding, the effect is difficult to characterize and does not affect the results for learning nor those for the differences in learning between the two age groups (all $F$ s for appropriate interactions $<1.3$, all $P$ s $>0.1$ ).

\section{Discussion}

The comparable performance of the two groups in both baseline phases suggests that our use of the stylus to prevent any age-linked motor

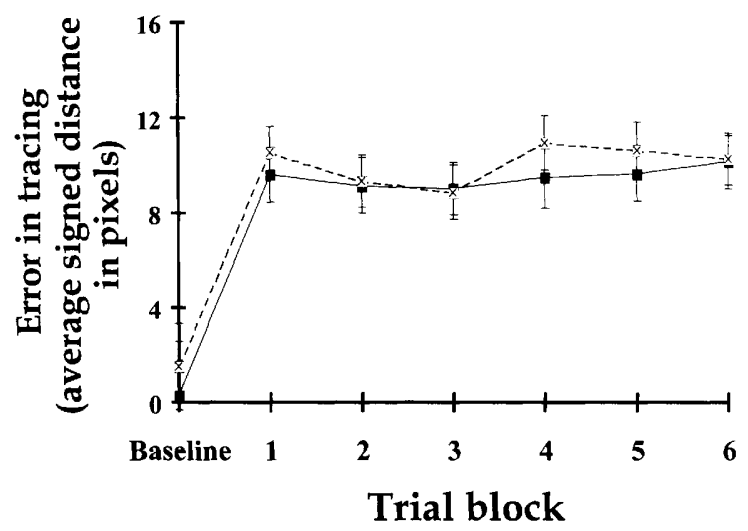

Figure 3: Mean adaptation for young $(\square)$ and older ( groups in the test phase, by block of test trials. Adaptation is measured as detailed in the text. Vertical error bars depict S.E.M.. performance effects was successful and that the presence or absence of feedback had no age-linked effect. In addition, this result strongly suggests that sensory acuity did not differ between the two groups; in fact, recent work has shown that any difference in proprioceptive acuity is likely to be in the direction of increased acuity with aging (Meeuwsen et al. 1993; Proteau et al. 1994). As Durkin et al. (1995) have pointed out, age-linked deficits in performance do not necessarily imply a deficit in learning. In investigations of the effect of aging on learning, it is optimal to use a task such as the one presented here in which baseline performance is comparable across age groups (thus suggesting that the level of cognitive load imposed is sufficiently low as to be within the range of ability of participants in all age groups).

Both groups showed adaptation to the transformation (i.e., a positive deviation in the test phase) that did not reach the maximum level of the transformation presented. This failure of adaptation to reach the maximum possible level, even over many trials, is in line with data from prism adaptation experiments (e.g., Welch 1978; Redding et al. 1985; Rossetti et al. 1993), and is consistent with other experiments in our laboratory (D.B. Willingham and N. Greenberg, unpubl.). Prism adaptation data in the literature has generally been interpreted as showing a gradual adaptation. However, the maximum level of adaptation in prism spectacle experiments is often seen after between 20 and 30 trials (Welch 1986), and so we believe that this apparent difference is simply a case of difference in the number of trials used before measurement of adaptation.

We predicted that the older participants would show a deficit in the training phase (because strategies could be used) but would show no deficit in the test phase. These predictions were confirmed. Thus, the data presented here support the suggestion that the effect of aging on motor skill learning is to reduce the resources available for formation of strategies. This pattern of findings also further supports the nonstrategic nature of the test phase task used, because any contamination by explicit, strategic learning would be expected to have introduced an age-linked performance deficit. Use of strategies in a training task has been shown previously to have little, if any, effect on implicit learning (Verdolini-Marston and Balota 1994), in line with our findings here. In a second study of the effects of strategy use on implicit learning, Redding and Wallace (1993) hypothesized that use of 


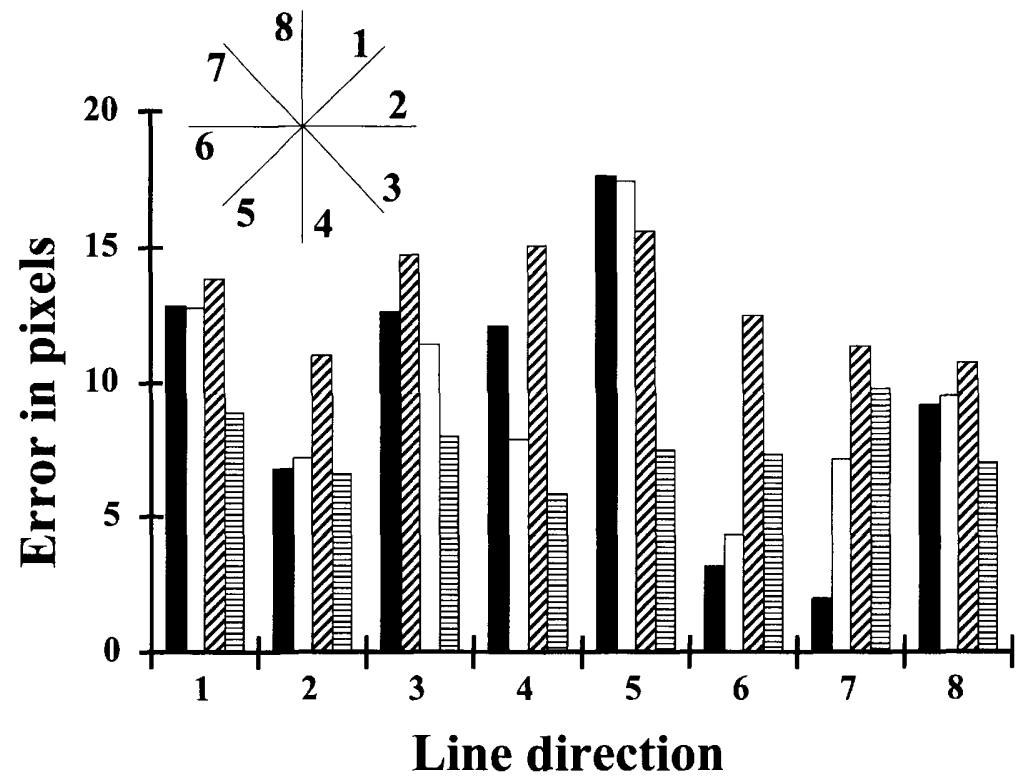

Figure 4: Mean errors for young and older subjects by line direction, across blocks, in both the training phase (young, horizontal-line bars; old, hatched bars) and the test phase (young, open bars; old, solid bars). effective strategies in an explicit task might reduce adaptation (owing to the reduced performance error in the explicit task leading to a reduced need for realignment of visual and proprioceptive mappings) but saw little, if any, such effect. Similarly, no such effect was seen in the present study.

It is important to remember that measures of learning in the two tasks used here are measures of the same learning of the same information (during the training phase). Thus, the present data cannot be explained by a simple age-related effect of information complexity on learning.

As far as we are aware, the effect of aging on perceptuomotor adaptation and negative aftereffect has not been investigated previously. One previous study (Canavan et al. 1990) tested both young and older participants on a prism spectacle pointing task in which visual feedback as to arm location was provided only at the end of each pointing. Canavan et al. do not discuss their data with respect to the effects of aging, but they found no difference in trials to criterion across age groups. Canavan et al.'s task would appear to be open to the use of strategies in a similar manner to the training phase of the present experiment. One possible explanation for the apparent discrepancy in results is that Canavan et al.'s task was considerably simpler than ours, and development of appropriate strategies may have placed an insufficient burden on cognitive resources to differentiate older participants from young controls. A limited number of previous studies have investigated the effect of aging on motor skill learning
(Ruch 1934; Gutman 1965; Wright and Payne 1985; Howard and Howard 1989, 1992) but have used tasks in which measurement of nonstrategic learning was not possible and/or in which young participants do not spontaneously discover strategies, such as SRT.

Our findings suggest that adaptation (at least in this paradigm) is not affected by any loss of attentional resources, such as occurs in aging (Salthouse 1988, 1990), as has been suggested previously (Redding et al. 1985). The question of whether, and under what circumstances, implicit learning requires attentional or other cognitive resources is as yet unresolved (Nissen and Bullemer 1987; Cohen et al. 1990; Frensch et al. 1994; Stadler 1995). One alternative possibility is that the adaptation task used here placed insufficient demand on cognitive resources to differentiate between young and older participants. However, lack of a requirement for significant cognitive resources in implicit perceptuomotor learning is supported by data from patients with Huntington's disease, who have reduced working memory capacity. Such patients show no deficit in adaptation compared with agematched controls although they show impairment in a training task similar to the one used here (Paulsen et al. 1993). Similarly, although the data on Alzheimer's patients is less clear, both Paulsen et al. (1993) and Weiner et al. (1983) find no reduction in adaptation in this condition, which has typically been viewed as reducing available cognitive resources. Further testing of young and older participants under conditions of additional cogni- 
tive load might provide the needed data to resolve this question.

The effect of aging on nonmotor skill learning has been suggested to be one of reduced perceptual organization (Hashtroudi et al. 1991), with older individuals performing poorly under conditions of limited data availability. The present study made no attempt to inhibit participants' perceptual input; this, taken together with the fact that both tasks measured learning from the same set of perceptual information yet gave different results for the effect of age on performance, makes it unlikely that the effect of aging on motor skill learning observed here was attributable to differences in perceptual organization. Similarly, the finding of no difference between groups in either of the baseline conditions argues strongly against our results being attributable to a difference in motor tracing ability or in ability to cope with the absence of feedback.

One possible explanation of the present results might have been an age-related difference in ability to alternate between the two tasks. If this were the case, one would expect to see a pattern of greater age-related error in early training trials within each block and, possibly, some pattern by position within block on test trials. However, examination of the data on a trial-by-trial basis shows no such pattern in either group. It is also possible that the two groups used different strategies rather than having different success in application of the same strategies. In one sense, such a finding would not alter our interpretation of the results, because it would still suggest an age-related deficit in the generation of useful strategies; nevertheless, the possibility remains. However, although no formal assessment of strategy use was conducted, conversations with participants after completion of the experiment confirmed that all members of both groups attempted to use strategies in the training phase and appeared to construct very similar strategies. Experiments currently underway, in which participants are provided with a strategy beforehand, will further investigate this point.

It is of interest to note that the level of adaptation displayed does not appear to be linked to performance in the training task: Adaptation achieves its maximum level after the first block of test trials, whereas training performance in both groups continues to improve across the training trial blocks. In this regard, it is important to note that the motor experience of the two groups was similar, as shown by their comparable lengths of line drawn. It has been suggested (Bedford 1995;
Willingham 1997) that unconscious motor learning occurs as a result of motor practice. If this is the case, the present data suggest that there is a limit to unconscious, nonstrategic motor learning that occurs well below the maximum possible performance level. Recent data (Redding and Wallace 1993 ) suggest that the relationship between training performance and test adaptation in prism spectacle tasks similar conceptually to those presented here, if any, is not a simple one, but it is possible that age-related differences in adaptation might be seen if some mechanism could be found to increase the apparent limits to such learning.

One could argue that it makes sense evolutionarily for there to be no deficit in adaptation with aging if, as has been suggested, the ecological basis for adaptation mechanisms is accommodation to an organism's changes in size with development (Bedford 1995); older humans are at least as likely as adults to experience changes in body shape and size (although the age at which such changes occur has altered markedly across evolutionary time as lifespan has changed, the fact of such changes in relatively old individuals has remained constant). The existence of such a basis for adaptation might be investigated by comparing the ease of adaptation to perturbed input from various body parts across age groups; the prediction would be that inputs from body areas prone to changes in size and/or shape at a given age would be more easily recalibrated.

\section{Acknowledgments}

We thank Nevin Greenberg for assistance with computer programming. This work was supported in part by the Virginia Center on Aging. The e-mail address of D.B.W. is willingham@virginia.edu.

The publication costs of this article were defrayed in part by payment of page charges. This article must therefore be hereby marked "advertisement" in accordance with 18 USC section 1734 solely to indicate this fact.

\section{References}

Bedford, F.L. 1993a. Perceptual and cognitive spatial learning. J. Exp. Psychol. Hum. Percep. Perform. 19: $517-530$. 30: $1-60$. 1993b. Perceptual learning. Psych. Learn. Motivation

\section{- 1995. Constraints on perceptual learning: Objects} and dimensions. Cognition 54: 253-297.

Canavan, A.G.M., R.E. Passingham, C.D. Marsden, N. Quinn, 
MOTOR SKILL LEARNING IN AGING

M. Wyke, and C.E. Polkey. 1990. Prism adaptation and other tasks involving spatial abilities in patients with Parkinson's disease, patients with frontal lobe lesions and patients with unilateral temporal lobectomies. Neuropsychologia 28: 969-984.

Cerella, J. 1990. Aging and information-processing rate. In Handbook of the psychology of aging (ed. J.E. Birren and K. Schaie), 3rd ed., pp. 201-221. Academic Press, New York, NY.

Cohen, A., R.I. Ivry, and S.W. Keele. 1990. Attention and structure in sequence learning. J. Exp. Psychol. Learn. Mem. \& $\operatorname{Cog} .16: 17-30$.

Craik, F.I.M. 1994. Memory changes in normal aging. Curr. Direct. Psychol. Sci. 5: 155-158.

Craik, F.I.M. and J.M. Jennings. 1992. Human Memory. In The handbook of aging and cognition (ed. F. Craik and T. Salthouse). Erlbaum, Hillsdale, NJ.

Cunningham, H.A. 1985. An Apple microcomputer-based laboratory system for the study of visual-motor behavior. Behav. Res. Methods Instrum. Comput. 17: 484-488.

1989. Aiming error under transformed spatial mappings suggests a structure for visual-motor maps. J. Exp. Psychol. Hum. Percep. Perform. 15: 493-506.

Durkin, M., L. Prescott, E. Furchtgott, J. Cantor, and D.A. Powell. 1995. Performance but not acquisition of skill learning is severely impaired in the elderly. Arch. Gerontol. Geriatr. 20: 167-183.

Fisk, A.D., N.D. McGee, and L.M. Giambra. 1988. The influence of age on consistent and varied semantic-category search performance. Psychol. Aging 3: 323-333.

Frensch, P.A., A. Buchener, and J. Lin. 1994. Implicit learning of unique and ambiguous serial transitions in the presence and absence of a distractor task. J. Exp. Psychol. Learn. Mem. \& Cog. 20: 567-584.

Gutman, G.M. 1965. The effects of age and extroversion on pursuit rotor reminiscence. J. Gerontol. 20: 346-350.

Harrington, D.L. and K.Y. Haaland. 1992. Skill learning in the elderly: Diminished implicit and explicit memory for a motor sequence. Psychol. Aging 7: 425-434.

Hashtroudi, S, L.D. Chrosniak, and B.L. Schwartz. 1991. Effects of aging on priming and skill learning. Psychol. Aging 6: $605-615$.

Howard, D.V. and J.H. Howard Jr. 1989. Age differences in learning serial patterns: Direct versus implicit measures. Psychol. Aging 4: 357-364.

1992. Adult age differences in the rate of learning serial patterns: Evidence from direct and indirect tests. Psychol. Aging 7: 232-241.
Light, L.L., D. La Voie, and R. Kennison. 1995. Repetition priming of nonwords in young and older adults. J. Exp. Psychol. Learn. Mem. \& Cog. 21: 327-346.

Meeuwsen, H.J., T.M. Sawicki, and G.E. Stelmach. 1993. Improved foot position sense as a result of repetitions in older adults. J. Gerontol. Psychol. Sci. 3: P137-P141.

Nissen, M.J. and P. Bullemer. 1987. Attentional requirements of learning: Evidence from performance measures. Cognit. Psychol. 19: 1-32.

Paulsen, J.S., N. Butters, D.P. Salmon, W.C. Heindel, and M.R. Swanson. 1993. Prism adaptation in Alzheimer's and Huntington's disease. Neuropsychology 7 : 73-81.

Proteau, L., I. Charest, and S. Chaput. 1994. Differential roles with aging of visual and proprioceptive afferent input for fine motor control. J. Gerontol. Psychol. Sci. 49: P100-P107.

Redding, G.M. and B. Wallace. 1978. Sources of "overadditivity" in prism adaptation. Percep. Psychophysics 20: 453-459.

1993. Adaptive coordination and alignment of eye and hand. J. Motor Behav. 25: 75-88.

Redding, G.M., S.E. Clark, and B. Wallace. 1985. Attention and prism adaptation. Cognit. Psychol. 17: 1-25.

Rossetti, Y., K. Koga, and T. Mano. 1993. Prismatic displacement of vision induces transient changes in the timing of eye-hand coordination. Percep. Psychophysics 54: 355-364.

Ruch, F.L. 1934. The differential effects of age upon human learning. J. Gen. Psychol. 11: 261-286.

Salthouse, T.A. 1988. Initiating the formalization of theories of cognitive aging. Psychol. Aging 3: 3-16.

1990. Working memory as a processing resource in cognitive aging. Dev. Rev. 10: 101-124.

Samuel, J.M.F. 1981. Individual differences in the interaction of vision and proprioception. In Intersensory perception and sensory integration (ed. R.D. Walk and H.L. Pick), pp. 374-398. Plenum, New York, NY.

Schacter, D.L. 1987. Implicit memory: History and current status. J. Exp. Psychol. Learn. Mem. \& Cog. 13: 501-518.

Stadler, M.A. 1995. The role of attention in implicit learning. J. Exp. Psychol. Learn. Mem. \& Cog. 21: 674-685.

Verdolini-Marston, K. and D.A. Balota. 1994. Role of elaborative and perceptual integrative processes in perceptual-motor performance. J. Exp. Psychol. Learn. Mem. \& Cog. 20: 739-749.

Wallace, B. and G.M. Redding. 1979. Additivity in prism adaptation as manifested in intermanual and interocular transfer. Percep. Psychophysics 25: 133-136. 


\section{McNay and Willingham}

Weiner, M.J., M. Hallett, and H.H. Funkenstein. 1983.

Adaptation to lateral displacement of vision in patients with lesions of the central nervous system. Neurology

33: $766-772$.

Welch, R.B. 1978. Perceptual modification. Academic Press, New York, NY.

1986. Adaptation of space perception. In Handbook of perception and human performance (ed. R.K. Boff, L. Kaufman, and J.P. Thomas), Vol. 1, pp. 24.1-24.45.

Wiley-Interscience, New York, NY.

Willingham, D.B. 1997. Systems of memory in the human brain. Neuron 18: 1-4.

1998. COBALT: A neuropsychological theory of motor skill learning. Psychol. Rev. (in press).

Wright, B.M. and R.B. Payne. 1985. Effects of aging on sex differences in psychomotor reminiscence and tracking proficiency. J. Gerontol. 40: 179-184.

Received July 7, 1997; accepted in revised form January 5, 1998. 


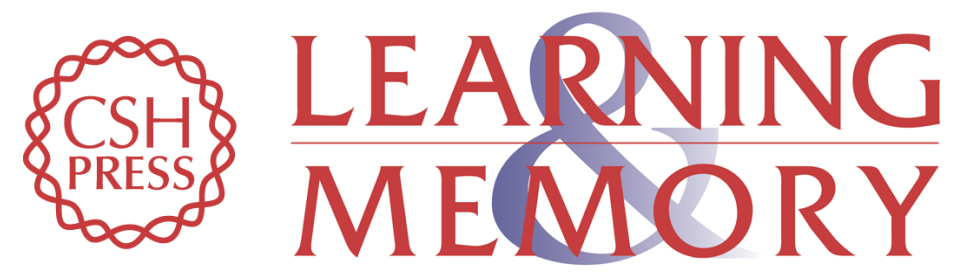

\section{Deficit in learning of a motor skill requiring strategy, but not of perceptuomotor recalibration, with aging.}

E C McNay and D B Willingham

Learn. Mem. 1998, 4:

Access the most recent version at doi:10.1101//m.4.5.411

\section{License}

Email Alerting

Service

Receive free email alerts when new articles cite this article - sign up in the box at the top right corner of the article or click here. 